\title{
Estado da arte da propagação \\ vegetativa por estaquia de espécies \\ arbustivo-arbóreas
}

REVISTA ELETRÔNICA

CIENTÍFICA DA UERGS

Sofia Foladori-Invernizzi

Universidade Federal do Paraná (UFPR).

E-mail: sofiafoladori@gmail.com, http://lattes.cnpq.br/26054I6746504376

Renata de Almeida Maggioni

Universidade Federal do Paraná (UFPR).

E-mail: renata.maggioni@ufpr.br, http://lattes.cnpq.br/275507II46084749

Katia Christina Zuffellato-Ribas

Universidade Federal do Paraná (UFPR).

E-mail: kazu@ufpr.br, http://lattes.cnpq.br//298025007। I 9630

ISSN 2448-0479. Submetido em: 20 maio 2020. Aceito: 19 nov. 2020. DOI: http://dx.doi.org/10.21674/2448-0479.71.50-63

\section{Resumo}

A propagação vegetativa por estaquia é uma técnica muito utilizada, por permitir a produção de mudas em espécies que possuem uma baixa taxa de germinação e viabilidade das sementes, além de permitir homogeneidade no plantio. Contudo, na propagação por estaquia, diversos fatores devem ser considerados, e há uma grande quantidade de variáveis a serem escolhidas. Assim, o objetivo deste trabalho foi realizar uma revisão sobre o estado da arte de espécies arbustivo-arbóreas propagadas pela técnica de estaquia comparando os resultados obtidos com diferentes estações do ano, tipo de estaca (herbácea, semilenhosa e lenhosa) e presença ou ausência de folhas nas estacas. A partir dessas características, buscou-se determinar uma tendência nos resultados, a fim de simplificar posteriores escolhas na realização de novos estudos com estaquia para espécies arbustivo-arbóreas. A busca foi realizada na base de dados da SCIELO e no portal de periódicos da CAPES, nos quais foram utilizados quatro conjuntos de palavras-chaves em inglês, sendo estes: "propagation and native species", "propagation and number of leaves", "propagation and season" e "propagation and types of cutting". Depois do refinamento e seleção dos artigos, chegou-se em 64 artigos para serem utilizados no estudo. Na revisão realizada, foi possível observar uma prevalência de resultados positivos quando utilizadas estacas herbáceas com folhas na primavera ou verão, sendo então estas variáveis recomendadas para a propagação de espécies arbóreas por estaquia.

Palavras-chave: Estaquia. Estação do ano. Tipo de estaca. Presença de folhas.

\section{Abstract}

\section{State of the art on the vegetative propagation of shrub-tree species}

Cutting is an extremely used and diffused propagation technique, as it allows to propagate plants with low rates of germination and viability of the seeds, in addition to bringing homogeneity to planting. During the process of cutting, to be able to obtain the best results, there are several factors and variables to be chosen from. Therefore, the objective of this study was to review the state of the art on the propagation by cutting of shrub-tree species, comparing results obtained by different seasons of the year, type of cutting (herbaceous, semi-hardwood and hardwood), and the presence or absence of leaves. From the analyzed characteristics, a tendency in the results was investigated, seeking to simplify future studies in the cutting area by reducing the choices of variables to be made. The literature search was done using four groups of keywords in english on 
the data bases of SCIELO and CAPES portal. The groups of keywords used were: "propagation and native species", "propagation and number of leaves", "propagation and season" and "propagation and types of cutting". After the search and refinement of the articles, 64 were selected for this study. The literature review showed the prevalence of positive results using herbaceous cuttings with leaves during spring or summer, being these variables recommended for the propagation via cutting of shrub-tree species.

Keywords: Cutting. Seasons of the year. Type of cutting. Presence of leaves.

\section{Resumen}

\section{Estado del arte de la propagación vegetativa por esquejes de especies arbóreas/arbustivas}

La propagación vegetativa por esquejes es una técnica muy difundida por permitir la producción de mudas en especies que tienen bajos índices de germinación y viabilidad de sus semillas, además de permitir una mayor homogeneidad en el plantío. Sin embargo, en la propagación por esquejes hay diversos factores que deben ser considerados y una diversidad de variables a elegir. De esta forma, el objetivo de este trabajo fue realizar una revisión sobre el estado del arte de especies arbóreas/arbustivas propagadas por la técnica de esqueje comparando los resultados obtenidos en diferentes estaciones del año, tipo de esquejes (herbáceo, semileñoso y leñoso) y la presencia o ausencia de hojas en los esquejes. A partir de estas características, se buscó determinar una tendencia en los resultados, visando simplificar decisiones posteriores en la realización de nuevos estudios con esquejes para especies del tipo arbóreas/arbustivas. La búsqueda fue realizada en la base de datos de SCIELO y en el portal de periódicos de la CAPES. En estos fueron utilizados cuatro conjuntos de palabras clave en inglés, siendo ellas: "propagation and native species", "propagation and number of leaves", "propagation and season" e "propagation and types of cutting". Después de seleccionar los artículos relevantes, 64 de ellos fueron utilizados para el estudio. En la revisión, fue posible observar una prevalencia de resultados positivos al utilizar esquejes herbáceos con hojas durante primavera o verano, siendo entonces estas las variables recomendadas para la propagación de especies arbóreas/arbustivas por esquejes.

Palabras clave: Esquejes. Estación del año. Tipo de esquejes. Presencia de hojas.

\section{Introdução}

A dificuldade de obtenção de mudas de espécies nativas está diretamente vinculada ao fato que estas geralmente possuem dormência nas sementes, dificultando sobremaneira a germinação (SANTOS et al., 2003). Diante disso, a propagação vegetativa apresenta-se como uma excelente alternativa para produção de mudas para fins ambientais em espécies com limitações na propagação seminal (CARPANEZZI; CARPANEZZI, 2006; OLIVEIRA; RIBEIRO, 20I3). Outra vantagem é a uniformidade no plantio, maior produtividade, baixo custo e a possível produção de mudas durante o ano todo (XAVIER, 2009; DIAS et al., 20 I2; WENDLING; STUEPP; ZUFFELLATO-RIBAS, 2016).

Entre as técnicas de propagação vegetativa, a estaquia é um método amplamente difundido, economicamente viável, de grande simplicidade e rapidez na execução, sendo uma técnica muito utilizada na produção de mudas florestais. A estaquia utiliza partes de uma planta matriz capazes de se desenvolverem em uma planta completa. Isso requer a formação de um novo sistema de raízes nas estacas. $O$ sucesso do enraizamento e da sobrevivência das estacas envolve diversos fatores, tanto endógenos, como balanço hormonal e idade, quanto exógenos, como a utilização de reguladores vegetais (ZUFFELLATO-RIBAS; RODRIGUES, 200 I; ZHANG et al., 20I6; HARTMANN et al., 20I8; STUEPP et al., 20I8).

A maturidade do material utilizado para a confecção das estacas é de grande importância devido a variações na concentração de auxinas endógenas, lignificação do tecido e acumulo de inibidores. A época do ano também é um dos fatores que pode afetar a indução do enraizamento adventício dos propágulos vegetais por alterarem as condições fisiológicas da planta matriz (ZUFFELLATO-RIBAS; RODRIGUES, 200 I; DIAS et al., 20I2; HARTMANN et al., 20I8). 
Assim, o presente estudo teve por objetivo realizar uma revisão bibliográfica sobre o estado da arte da propagação vegetativa por estaquia de espécies arbustivo-arbóreas para determinar quais as melhores variáveis para o processo de estaquia. As variáveis analisadas consistiram na presença ou ausência de folhas, tipo de estaca e estação do ano para instalação.

\section{Material e Métodos}

Para a realização da revisão bibliográfica sobre o estado da arte da propagação vegetativa por estaquia de espécies arbustivo-arbóreas foram utilizadas duas bases de dados: a SCIELO (https://www.scielo.br/) e o portal de periódicos da CAPES (https://www.periodicos.capes.gov.br/). Nos dois casos foram utilizados quatro conjuntos de palavras-chave em inglês, sendo estes "propagation and native species" (propagação e espécie nativa), "propagation and number of leaves" (propagação e número de folhas), "propagation and season" (propagação e estação), "propagation and types of cutting" (propagação e tipos de estaca). A busca nas bases de dados foi realizada durante janeiro de 2020. Não foi realizado nenhum recorte temporal para os resultados obtidos.

Dos resultados obtidos nas plataformas da SCIELO e do portal de periódicos da CAPES a partir das palavras-chave, foram selecionados apenas artigos de propagação por estaquia de espécies arbustivo-arbóreas. Foram selecionados artigos de estaquia e miniestaquia para as variáveis número de folhas e estação do ano. Contudo, para a variável tipo de estaca apenas foram selecionados trabalhos de estaquia com ramos herbáceos, semilenhosos e/ou lenhosos. Não foram consideradas espécies herbáceas, epífitas e cactos. Todas as plantas do tipo arbustivo-arbóreas, independente da espécie e do local de realização do estudo foram consideradas.

Para refinar a busca, foram aceitos somente artigos em português, inglês e espanhol. Além disso, em algumas das buscas, devido à quantidade de artigos obtidos, foi realizado um refinamento em subtópicos. Em todos os casos, no portal da CAPES foi selecionado o grande tema "agricultura e florestamento". Para o conjunto de palavras-chave propagação e espécie nativa, na base SCIELO não foi realizado algum refinamento e no portal da CAPES foram escolhidos os subtópicos: propagação, propagação vegetativa e propagação de plantas. Para propagação e número de folhas, na base SCIELO foram selecionados os temas: agricultura, horticultura, agronomia e ciências das plantas; não foi realizado nenhum refinamento adicional no portal da CAPES além do grande tema. No grupo propagação e estação na base SCIELO não foi realizado refinamento e no portal da CAPES foi selecionado o subtópico propagação e retirados os tópicos: germinação, sementes, precipitação, oceanografia, meteorologia, marinho, chuva, física, propagação de ondas e atmosfera. No último conjunto, propagação e tipos de estaca, não foi realizado nenhum refinamento na base SCIELO; já no portal da CAPES foram selecionados: estaquia, propagação e propagação vegetativa, e retirados os tópicos: cultura de tecido, diversidade genética, doenças de plantas e patologias de plantas.

Uma vez selecionados os artigos nas bases de dados e realizada a leitura, os resultados utilizados no trabaIho foram separados em três quadros conforme as três variáveis de interesse a serem comparadas e a espécie de cada estudo. Sendo assim, foi realizado um quadro para os artigos que tratavam de diferenças no número de folhas; outro para artigos comparando diferentes tipos de estaca; e um terceiro para os artigos vinculados a diferentes épocas do ano.

\section{Resultados e Discussões}

Na revisão bibliográfica foram obtidos 552 resultados na base SCIELO, dos quais 50 foram selecionados. Desses 50 selecionados, 24 foram repetidos em várias buscas, tanto na SCIELO quanto na CAPES. No portal da CAPES foram obtidos I78I resultados, dos quais 55 foram selecionados, e desses 55, 20 deles repetidos em várias buscas (Quadro I). No total, excluídas as repetições nas bases de dados, foram utilizados 64 trabaIhos que estão referenciados nos respectivos quadros dependendo das variáveis analisadas em cada trabalho (Quadros 2, 3 e 4). 
Quadro I - Resultados da busca na base de dados SCIELO e no portal de periódicos da CAPES

\begin{tabular}{|c|c|c|c|}
\hline Base de dados & Palavras-chave & Resultados & Resultados utilizados \\
\hline \multirow{4}{*}{ SCIELO } & Propagation and native species & 196 & 12 \\
\hline & Propagation and number of leaves & 200 & 10 \\
\hline & Propagation and season & 73 & 11 \\
\hline & Propagation and types of cuttings & 83 & 17 \\
\hline \multirow{4}{*}{ CAPES } & Propagation and native species & 635 & 13 \\
\hline & Propagation and number of leaves & 316 & 8 \\
\hline & Propagation and season & 483 & 10 \\
\hline & Propagation and types of cuttings & 347 & 24 \\
\hline
\end{tabular}

Como já mencionado, os resultados obtidos foram separados em três quadros conforme as três variáveis: número de folhas (Quadro 2), tipo de estaca (Quadro 3) e época do ano (Quadro 4).

A presença de folhas nas estacas confeccionadas afeta a taxa de enraizamento, por serem fontes de auxina, cofatores e fotoassimilados necessários à rizogênese. Os carboidratos presentes nas folhas são translocados em direção à base da estaca, atuando como importantes fontes de energia, macromoléculas e elementos estruturais para a formação das raízes, mesmo sem induzir diretamente a formação destas. Contudo, a manutenção de um grande número de folhas ou de uma grande área foliar pode aumentar excessivamente a taxa de transpiração, provocando a morte da estaca (ZUFFELLATO-RIBAS; RODRIGUES, 200 I; HARTMANN et al., 20I8).

Em relação a estacas com diferentes números de folhas (Quadro 2 e Gráfico IA), foram comparados I7 trabalhos envolvendo I5 espécies. Das I5 espécies 13 tiveram melhores resultados de enraizamento com o aumento do número de folhas, sendo que para algumas espécies a manutenção das folhas também levou a meIhores resultados em outras variáveis, como sobrevivência, calogênese, número e volume de raízes. A espécie Olea europaea foi utilizada em três trabalhos, sendo que em todos apresentou melhores resultados com folhas, aparentando ser uma variável importante para a espécie (PIO et al., 2005; PENSO et al., 2016; PORFíRIO et al., 2016). Apenas duas espécies das 15 comparadas, Punica granatum e Paulownia fortunei, apresentaram melhores resultados em estacas sem folhas (PAIVA et al., 20I5; STUEPP et al., 20I5a).

Quadro 2 - Resultados que comparam a confecção de estacas com diferentes números de folhas para diferentes espécies com base nos artigos da revisão bibliográfica sobre estaquia de espécies arbustivo-arbóreas

\begin{tabular}{|c|c|c|c|}
\hline Espécie & Número de folhas & Resultados & Referência \\
\hline $\begin{array}{l}\text { Azadirachta indica A. } \\
\text { Juss. }\end{array}$ & $\begin{array}{l}\text { Área foliar de } 30,50 \text { e } \\
\qquad 100 \%\end{array}$ & $\begin{array}{c}\text { Sem diferença significativa no número de } \\
\text { estacas enraizadas. } 100 \% \text { área: melhor } \\
\text { enraizamento }\end{array}$ & (KAMALUDDIN; ALI, 1996) \\
\hline $\begin{array}{l}\text { Caryocar brasiliense } \\
\text { Camb. }\end{array}$ & 0,6 pela metade, 6 & $\begin{array}{c}\text { Sem folhas: } 100 \% \text { de mortalidade. Com } \\
\text { folhas: melhor enraizamento, sobrevivência e } \\
\text { calogênese. }\end{array}$ & (GUIMARÃES et al., 2019) \\
\hline $\begin{array}{l}\text { Couepia edulis (Prance) } \\
\text { Prance }\end{array}$ & 0 , pela metade, inteiras & $\begin{array}{l}\text { Estacas com folhas inteiras e reduzidas pela } \\
\text { metade tiveram melhores resultados. }\end{array}$ & $\begin{array}{l}\text { (LEANDRO; YUYAMA, } \\
\text { 2008) }\end{array}$ \\
\hline $\begin{array}{l}\text { Dovyalis hebecarpa } \\
\text { (Gardner) } \\
\text { Warb. }\end{array}$ & 2,4 e 6 & $\begin{array}{l}\text { Melhor resultado com a manutenção de } \\
\text { quatro folhas. }\end{array}$ & (RINALDI et al., 20I8) \\
\hline Drymis brasiliensis Miers & $0,1,2$ & $\begin{array}{l}\text { Melhor enraizamento e menor mortalidade } \\
\text { com duas folhas por estaca. }\end{array}$ & (ZEM et al., 20I6) \\
\hline $\begin{array}{l}\text { Lippia alba (Mill) N. E. } \\
\text { Brown }\end{array}$ & $0,2,4$ & $\begin{array}{l}\text { Estacas com quatro folhas tiveram maior } \\
\text { número e volume de raízes, seguidas das com } \\
\text { duas folhas. }\end{array}$ & (BIASI; COSTA, 2003) \\
\hline Litchi chinensis Sonn. & $0,1,2,3,4$ folíolos & $\begin{array}{l}\text { O aumento no número de folíolos foi } \\
\text { vantajoso para todas as variáveis avaliadas. }\end{array}$ & (ALVES et al., 2016) \\
\hline Malpighia glabra L. & 0, I par, 2 pares & $\begin{array}{l}\text { Estacas sem folhas não enraizaram. Melhores } \\
\text { resultados com dois pares de folhas. }\end{array}$ & (GONTIJO et al., 2003) \\
\hline
\end{tabular}


Quadro 2 - Resultados que comparam a confecção de estacas com diferentes números de folhas para diferentes espécies com base nos artigos da revisão bibliográfica sobre estaquia de espécies arbustivo-arbóreas

\begin{tabular}{|c|c|c|c|}
\hline Espécie & Número de folhas & Resultados & Referência \\
\hline Olea europaea L. & 0,1 par, 2 pares & $\begin{array}{l}\text { Melhores resultados com folhas. Pio (2005): } \\
\text { Maior enraizamento em estacas com dois } \\
\text { pares de folhas. Maior número de brotações } \\
\text { em estacas sem folhas. Penso (2016): } \\
\text { Melhores resultados com pelo menos um } \\
\text { par de folhas. Porfírio (2006): revisão sem } \\
\text { resultados consistentes. Em geral, melhores } \\
\text { resultados com folhas. }\end{array}$ & $\begin{array}{c}\text { (PIO et al., 2005); } \\
\text { (PENSO et al., 2016); } \\
\text { (PORFÍRIO et al., 2016) }\end{array}$ \\
\hline $\begin{array}{c}\text { Paratecoma peroba } \\
\text { (Record \& Mell) Kuhlm. }\end{array}$ & $\begin{array}{l}\text { Área foliar de } 50 \text { e } \\
100 \%\end{array}$ & $\begin{array}{l}\text { Manutenção de toda a área foliar promoveu } \\
\text { um melhor sistema radicial, com maior } \\
\text { superfície, área e volume das raízes. }\end{array}$ & (ARAÚJO et al., 2019) \\
\hline $\begin{array}{l}\text { Paulownia fortunei } \\
\text { (Seem.) Hemsl. var. } \\
\text { mikado }\end{array}$ & 0,2 reduzidas & Melhor resultado sem folhas. & (STUEPP et al., 20।5a) \\
\hline $\begin{array}{l}\text { Plukenetia polyadenia } \\
\text { Müll.Arg. }\end{array}$ & $\begin{array}{l}\text { Área foliar de } 25,50 \text { e } \\
75 \%\end{array}$ & $\begin{array}{c}\text { Estacas com área foliar de } 50 \% \text { tiveram } \\
\text { melhores resultados. }\end{array}$ & (SOLIS et al., 2016) \\
\hline Punica granatum L. & 0,1 & $\begin{array}{l}\text { Melhor resultado sem folhas (com incisão na } \\
\text { base). }\end{array}$ & (PAIVA et al., 20I5) \\
\hline Rubus spp. & Sem e com & Maior enraizamento em estacas com folhas. & (VIGNOLO et al., 20I4) \\
\hline $\begin{array}{l}\text { Xylopia aethiopicato } \\
\text { (Dunal) A.Rich. }\end{array}$ & $0,1-2,3-4$ & $\begin{array}{l}\text { Melhores resultados de enraizamento com } \\
\text { três-quatro folhas. }\end{array}$ & $\begin{array}{l}\text { (MBIBONG; KANMEGNE; } \\
\text { FOTSO, 2019) }\end{array}$ \\
\hline
\end{tabular}

\section{Gráfico I -}

Resultados das diferentes espécies para as variáveis analisadas com base nos artigos da revisão bibliográfica sobre estaquia de espécies arbustivo-arbóreas. A. estacas com folhas e sem folhas ( 15 espécies); B. estacas herbáceas, lenhosas e semilenhosas ( 24 espécies); e C. diferentes estações do ano (40 espécies)

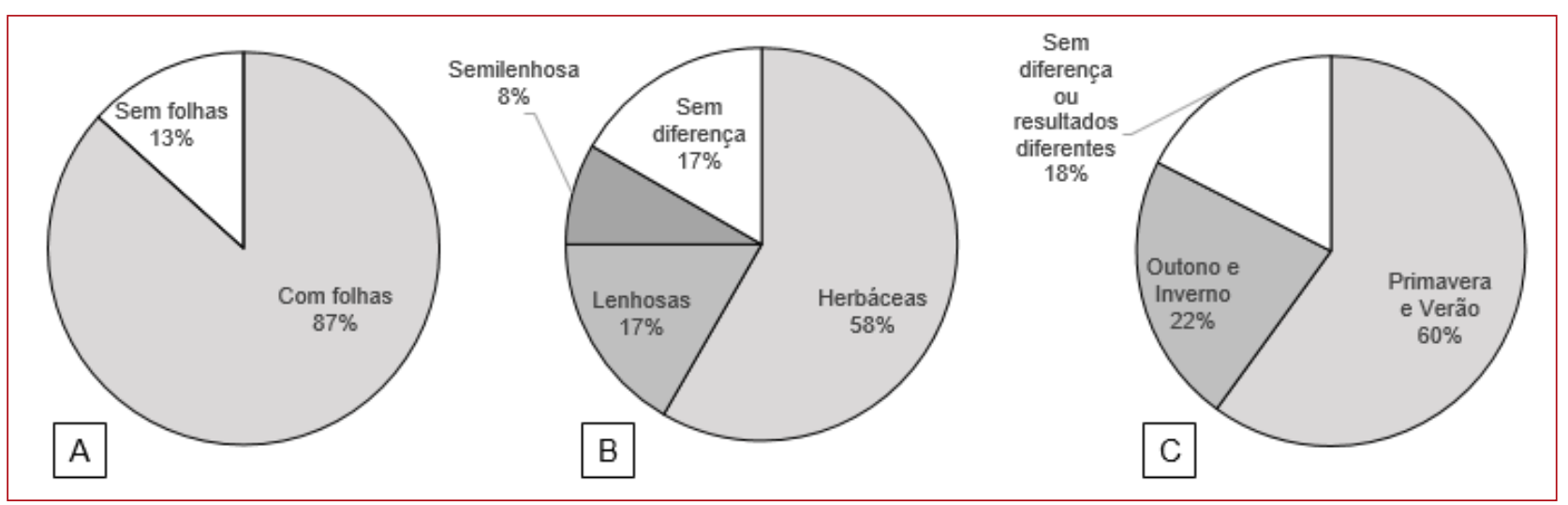

Em um artigo, utilizando a espécie Plukenetia polyadenia, foram obtidos melhores resultados com $50 \%$ da área foliar, quando comparado com 75\% (SOLIS et al., 2016). Rinaldi et al. (20I8), trabalhando com Dovyalis hebecarpa obtiveram melhores resultados mantendo quatro folhas, ao invés de duas ou seis. Estes resultados poderiam ser explicados tanto pela maior transpiração devido a uma maior área foliar, quanto ao efeito "guarda-chuva", quando folhas muito grandes impedirem a entrada de água por nebulização intermitente nos tubetes na casa de vegetação (BEYL; TRIGIANO, 20I5). A vantagem das folhas, como discutido anteriormente, está relacionada com a presença de compostos que facilitam o enraizamento, como carboidratos, proteínas e cofatores do enraizamento (HARTMANN et al., 20I8).

A lignificação do tecido é outro fator determinante no processo rizogênico. No Quadro 3 (Gráfico IB) comparou-se os tipos de estacas quanto ao nível de lignificação do tecido, que pode ser herbáceo, semilenhoso ou lenhoso. Dos 20 artigos comparados com um total de 24 espécies estudadas, quatro espécies, Campomanesia adamantium, Dalbergia sissoo, Malvaviscus arboreus e Senecio praecox, apresentaram melhores resultados com estacas lenhosas (PURI; VERMA, 1996; LOSS et al., 2009; RAMOS-PALACIOS et al., 20I2; MARTINS et al., 20I5) e em quatro espécies, Buddleja cordata, Citrus limettioides, Dodonaea viscosa e Ficus ca- 
rica, não houve diferença entre os tipos de materiais utilizados (RAMOS-PALACIOS et al., 20 I2; SOUSA et al., 2013; HUSSAIN et al., 2017). Singh e Rawat (2017) compararam estacas lenhosas e semilenhosas de Zanthoxylum armatum, obtendo melhores resultados com estacas semilenhosas, indicando que materiais mais jovens são mais propícios ao enraizamento. Biazatti e colaboradores (20l8), trabalhando com Psidium cattleianum e comparando estacas herbáceas e semilenhosas obtiveram enraizamento apenas com estacas semilenhosas, indicando que estacas muito tenras podem não enraizar. As demais 14 espécies obtiveram melhores resultados com estacas herbáceas.

Quadro 3 - Resultados que comparam os tipos de estacas para diferentes espécies com base nos artigos da revisão bibliográfica sobre estaquia de espécies arbustivo-arbóreas

\begin{tabular}{cc}
\hline Espécie & Tipo de estaca \\
\hline $\begin{array}{c}\text { Aloysia gratissima (Gillies \& } \\
\text { Hook.) Tronc. }\end{array}$ & Herbácea e semilenhosa \\
$\begin{array}{c}\text { Anadenanthera macrocarpa } \\
\text { (Benth.) Brenan. }\end{array}$ & Herbácea e semilenhosa \\
Buddleja cordata HBK. & Herbácea e lignificada \\
$\begin{array}{c}\text { Calophyllum brasiliense } \\
\text { Cambess. }\end{array}$ & Herbácea e lenhosa \\
$\begin{array}{c}\text { Campomanesia adamantium } \\
\text { (Cambess) O. Berg. }\end{array}$ & Herbácea e lenhosa
\end{tabular}

Citrus limettioides Tanaka. Herbácea e semilenhosa

[Citrus paradisi

MacFaden $\times$ Poncirus trifoliata (L.) Raf.] Couepia edulis (Prance)
Prance.

Dalbergia sissoo Roxb.

Disterigma alaternoides (Kunth) Nied.

Dodonaea viscosa Jacq.

Drimys brasiliensis Miers.

Erythrina falcata Benth.

Ficus carica L.

Macleania rupestris Kunth A.C. Sm.

Malvaviscus arboreus Cav.

Plinia cauliflora (Mart) Kausel.

Plinia jaboticaba (Vell.) Berg.

Psidium cattleianum sabine

Pterocarpus santalinoides L' Herit.

Rubus spp.
Herbácea, semilenhosa e lenhosa

Herbáceas e semilenhosas (sem folhas)

Herbácea e lenhosa

Herbácea e lenhosa

Herbácea e lignificada

Herbácea e semilenhosas

Herbácea e semilenhosa

(de mudas)

Herbácea e lenhosa

Herbácea, semilenhosa e lenhosa

Herbácea, semilenhosa e lenhosa

Revisão

Revisão

Herbácea e semilenhosas

Herbácea e semilenhosa

Herbácea e semilenhosa
Estacas herbáceas tiveram melhor enraizamento (quase 100\%).

Estacas herbáceas apresentaram maiores taxas de enraizamento.

Sem diferença entre os tipos de estaca.

Estacas herbáceas tiveram valores mais altos de enraizamento.

Estacas lenhosas são recomendadas.

Maior número de raízes em estacas herbáceas.

Estacas herbáceas apresentaram melhores resultados de propagação.

Maior enraizamento em estacas herbáceas com folhas inteiras e pela metade.

Estacas lenhosas tiveram melhores resultados.

Estacas jovens herbáceas tiveram melhores resultados.

Sem diferença entre os tipos de estaca.

Estacas herbáceas tiveram um melhor enraizamento.

Estacas herbáceas enraizaram e semilenhosas não. Melhores resultados em estacas de mudas.

Sem diferença entre o tipo de estaca.

Estacas herbáceas e semilenhosas são mais indicadas para a propagação da espécie.

Estacas lenhosas tiveram o melhor resultado na propagação da espécie.

Estacas herbáceas possuem melhores resultados no enraizamento.

Estacas herbáceas possuem melhores resultados no enraizamento.

Estacas herbáceas não enraizaram. Estacas semilenhosas enraizaram.

Melhores resultados com estacas herbáceas.

Estacas herbáceas e semilenhosas tiveram bons resultados.
Referência

(SANTOS et al., 2009)

(DIAS et al., 2015)

(RAMOS-PALACIOS et al., 20I2)

(CIRIELLO; MORI, 20I5)

(MARTINS et al., 2015)

(HUSSAIN, 2016)

(MOURÃO FILHO;

GIRARDI; THADEU

ZARATE DO

COUTO, 2019)

(LEANDRO;

YUYAMA, 2008)

(PURI; VERMA, 1996)

(MAGNITSKIY;

LIGARRETO;

LANCHEROS, 20II)

(RAMOS-PALACIOS

et al., 2012)

(ZEM et al., 20I5a)

(NEVES et al., 2006)

(SOUSA et al., 2013)

(VELOZA et al., 20।4)

(LOSS et al., 2009)

(SILVA et al., 2019)

(SILVA et al., 2019)

(BIAZATTI et al., 2018)

(KY-DEMBELE et al., 2016)

(HUSSAIN et al., 2017) 


\begin{tabular}{|c|c|c|c|}
\hline Espécie & Tipo de estaca & Resultados & Referência \\
\hline Senecio praecox D.C. & Herbácea e lignificada & Melhores resultados com estacas lignificadas. & $\begin{array}{l}\text { (RAMOS-PALACIOS } \\
\text { et al., 20I2) }\end{array}$ \\
\hline $\begin{array}{l}\text { Vaccinium floribundum } \\
\text { Kunth. }\end{array}$ & Herbácea e lenhosa & $\begin{array}{c}\text { Estacas jovens herbáceas tiveram melhores } \\
\text { resultados. }\end{array}$ & $\begin{array}{c}\text { (MAGNITSKIY; } \\
\text { LIGARRETO; } \\
\text { LANCHEROS, 20I I) }\end{array}$ \\
\hline Zanthoxylum armatum DC. & Semilenhosa e lenhosa & $\begin{array}{l}\text { Melhores resultados com estacas } \\
\text { semilenhosas. }\end{array}$ & $\begin{array}{c}\text { (SINGH; RAWAT, } \\
2017)\end{array}$ \\
\hline
\end{tabular}

Observa-se que os resultados para diferentes tipos de estaca são diversos e variam muito conforme a espécie. Contudo, é possível indicar uma tendência de melhores resultados utilizando estacas herbáceas para a multiplicação vegetativa por estaquia de espécies arbustivo-arbóreas.

O enraizamento dos ramos herbáceos está relacionado com a maior concentração de auxinas endógenas. Além disso, tecidos menos lignificados auxiliam no processo de enraizamento por não criar uma barreira anatômica para a emissão da raiz. Contudo, em alguns casos, estacas herbáceas podem ser muito tenras, diminuindo a eficiência da propagação devido aos baixos níveis de biossíntese e altas taxas de desidratação do tecido. Estacas com características lenhosas podem ser mais vantajosas em alguns casos, pois reduzem a desidratação do material, contudo, podem acumular inibidores de enraizamento (LEAKEY, 2004; XAVIER, 2009; HARTMANN et al., 2018).

No Quadro 4 observa-se o efeito das diferentes épocas do ano na propagação vegetativa. Pode-se notar uma tendência à melhor propagação durante as épocas de primavera e verão, dado que 24 das 40 espécies comparadas apresentam resultados mais promissores nessas estações (Gráfico IC). Cameron (2003), não obteve diferença entre as estações com Corylus avellana. Já Andrade (20I7), com Nephelium lappaceum, obteve $100 \%$ de mortalidade em todas as estações. A época do ano é um dos fatores que pode afetar a indução do enraizamento adventício pois as variações ambientais podem influenciar as condições fisiológicas da planta-matriz (ZUFFELLATO-RIBAS; RODRIGUES, 200I; HARTMANN et al., 20I8). Assim, a melhor época para coleta de material para a propagação por estaquia, segundo o presente estudo do estado da arte, é durante a primavera, seguido do verão para estacas de espécies arbustivo-arbóreas.

Quadro 4 - Resultados que comparam as épocas do ano para diferentes espécies com base nos artigos da revisão bibliográfica sobre estaquia de espécies arbustivo-arbóreas

\begin{tabular}{|c|c|c|c|}
\hline Espécie & Época do ano & Resultados & Referência \\
\hline Allamanda cathartica $L$. & O-I e I-P & $\begin{array}{l}\text { Melhores resultados no outono- } \\
\text { inverno. }\end{array}$ & $\begin{array}{l}\text { (KETTENHUBER; SOUSA; } \\
\text { SUTILI, 2019) }\end{array}$ \\
\hline $\begin{array}{l}\text { Boswellia papyrifera } \\
\text { (Del) Hochst. }\end{array}$ & $\mathrm{I}(02)$ e $\mathrm{P}(03,04$ e 05$)$ *HN & $\begin{array}{c}\text { Melhores resultados no inverno }(02) \\
\text { e na primavera }(03)\end{array}$ & (HAILE et al., 20II) \\
\hline $\begin{array}{c}\text { Campomanesia } \\
\text { adamantium (Cambess) } \\
\text { O. Berg. }\end{array}$ & $\mathrm{V}(02), \mathrm{O}(05)$ e $\mathrm{P}(\mathrm{I} 2)$ & $\begin{array}{l}\text { A melhor época foi durante o outono } \\
\text { (maio). }\end{array}$ & (MARTINS et al., 20I5) \\
\hline $\begin{array}{l}\text { Casearia sylvestris } \\
\text { Swartz. }\end{array}$ & Quatro estações & Melhores resultados na primavera. & (SPANDRE et al., 20I2) \\
\hline $\begin{array}{l}\text { Cephalanthus glabratus } \\
\text { (Spreng.) K.Schum. }\end{array}$ & O-I e I-P & Melhores resultados na primavera. & $\begin{array}{l}\text { (KETTENHUBER; SOUSA; } \\
\text { SUTILI, 2019) }\end{array}$ \\
\hline Citrus spp. & $\mathrm{O}$ e $\mathrm{P}$ & $\begin{array}{c}\text { O enraizamento ficou próximo a } \\
100 \% \text { na primavera. }\end{array}$ & $\begin{array}{l}\text { (SARMIENTO; SOUZA; } \\
\text { SCHWARZ, 2016) }\end{array}$ \\
\hline $\begin{array}{l}\text { Corylus avellana cv. } \\
\text { Aurea. }\end{array}$ & $\mathrm{P}(06)$ e $\mathrm{V}(07 ; 09) * \mathrm{HN}$ & Sem influência da época do ano. & (CAMERON et al., 2003) \\
\hline Dalbergia sissoo Roxb. & $\mathrm{I}$, monção e $\mathrm{P}$ & $\begin{array}{l}\text { A melhor época foi durante a } \\
\text { primavera (estação chuvosa). }\end{array}$ & (PURI; VERMA, 1996) \\
\hline
\end{tabular}




\begin{tabular}{|c|c|c|c|}
\hline Espécie & Época do ano & Resultados & Referência \\
\hline $\begin{array}{l}\text { Drimys brasiliensis } \\
\text { Miers. }\end{array}$ & $\begin{array}{l}\text { V e I (ZEM et al., 20I5a); } \\
\text { Quatro estações (ZEM et } \\
\quad \text { al., 20I5b) }\end{array}$ & $\begin{array}{c}\text { Resultados variados. Melhores } \\
\text { resultados no verão Zem (20 I 5a), e } \\
\text { no inverno Zem (20 I } 5 b) \text {. }\end{array}$ & $\begin{array}{l}\text { (ZEM et al., 20I5a); } \\
\text { (ZEM et al., 20I5b) }\end{array}$ \\
\hline Epacris impressa Labill. & Quatro estações & $\begin{array}{l}\text { Melhor enraizamento durante o } \\
\text { verão. }\end{array}$ & (THOMPSON, I986) \\
\hline Erythrina falcata Benth. & Quatro estações & $\begin{array}{l}\text { Maior enraizamento durante a } \\
\text { primavera. }\end{array}$ & (NEVES et al., 2006) \\
\hline $\begin{array}{l}\text { Escallonia bífida Link \& } \\
\text { Otto. }\end{array}$ & O-I e I-P & $\begin{array}{c}\text { Enraizamento somente durante a } \\
\text { primavera. }\end{array}$ & $\begin{array}{l}\text { (KETTENHUBER; SOUSA; } \\
\text { SUTILI, 2019) }\end{array}$ \\
\hline $\begin{array}{l}\text { Ficus binnendijkii } \\
\text { 'Amstel Queen' }\end{array}$ & $\mathrm{P}-\mathrm{V}(06)$ e V $(09) * \mathrm{HN}$ & $\begin{array}{l}\text { Melhores resultados durante o verão } \\
(09) .\end{array}$ & (BABAIE et al., 20I4) \\
\hline Ficus carica L. & $\begin{array}{c}\text { O-I (06), I (07 e 08) e I-P } \\
\text { (09) (SOUZA et al., 20 I 9); } \\
\text { I (07, 08) (NAVA et al., } \\
20 \text { I } 4) ; \\
\text { V (0I), O (05), I-P (09) } \\
\text { (PAULA et al., 2009) }\end{array}$ & $\begin{array}{l}\text { Resultados variados. Melhores } \\
\text { resultados no inverno: em agosto } \\
\text { (SOUZA et al. 20I9) e julho (NAVA } \\
\text { et al. 20I4). Paula et al. (2009): } \\
\text { melhores resultados no final de } \\
\text { outono, início da primavera (09) e no } \\
\text { verão (0I). }\end{array}$ & $\begin{array}{l}\text { (SOUZA et al., 2019); } \\
\text { (NAVA et al., 20I4); } \\
\text { (PAULA et al., 2009) }\end{array}$ \\
\hline $\begin{array}{l}\text { Ilex paraguariensis A. } \\
\text { St.-Hil. }\end{array}$ & Quatro estações & $\begin{array}{l}\text { Melhor enraizamento no outono, } \\
\text { seguido da primavera e inverno. }\end{array}$ & (STUEPP et al., 20I7) \\
\hline $\begin{array}{l}\text { Juniperus chinensis var. } \\
\text { kaizuka }\end{array}$ & $\mathrm{O}, \mathrm{I}$ e $\mathrm{P}$ & $\begin{array}{l}\text { Melhores resultados de } \\
\text { enraizamento na primavera. }\end{array}$ & (FRAGOSO et al., 20I5) \\
\hline $\begin{array}{l}\text { Ludwigia elegans } \\
\text { Thorell. }\end{array}$ & O-I e I-P & $\begin{array}{c}\text { Sem diferença significativa. Melhores } \\
\text { resultados na primavera. }\end{array}$ & $\begin{array}{l}\text { (KETTENHUBER; SOUSA; } \\
\text { SUTILI, 20I9) }\end{array}$ \\
\hline $\begin{array}{l}\text { Maytenus muelleri } \\
\text { Schwacke }\end{array}$ & $\mathrm{P}, \mathrm{V}, \mathrm{O}, \mathrm{I}$ & Melhores resultados no verão. & (LIMA et al., 20I I) \\
\hline Myrtus communis $\mathrm{L}$. & $\begin{array}{l}\text { I }(01,02,03), \mathrm{P}(04,06), \mathrm{V} \\
(08) \text { e O }(10,12) \text { *HN }\end{array}$ & $\begin{array}{l}\text { Enraizamento foi melhor no inverno } \\
\text { (dezembro, janeiro e fevereiro). }\end{array}$ & $\begin{array}{c}\text { (KLEIN; COHEN; HEBBE, } \\
\text { 2000) }\end{array}$ \\
\hline $\begin{array}{l}\text { Nephelium } \\
\text { lappaceum L. }\end{array}$ & Quatro estações & $\begin{array}{l}\text { 100\% de mortalidade em todas as } \\
\text { estações do ano. }\end{array}$ & (ANDRADE et al., 20I7) \\
\hline Olea europaea L. & $\begin{array}{c}\text { V-O (03), O (06), I-P (09) } \\
\text { e P-V (09) (PENSO et al., } \\
2016) ; \\
\text { O (04, 05), O-I (06) e I } \\
\text { (07 e 08) (OLIVEIRA et al., } \\
\text { 2009); } \\
\text { Revisão (PORFíRIO et al., } \\
2016)\end{array}$ & $\begin{array}{l}\text { Resultados variados. Penso ( } 2016) \text { : } \\
\text { melhores resultados no outono (07) } \\
\text { e inverno-primavera (09). Oliveira } \\
\text { e al. }(2009) \text { : melhores resultados no } \\
\text { início do outono (04). Porfírio et al. } \\
\text { (20।6): sem resultados consistentes, } \\
\text { melhores resultados no verão. }\end{array}$ & $\begin{array}{l}\text { (PENSO et al., 2016); } \\
\text { (OLIVEIRA et al., 2009); } \\
\text { (PORFÍRIO et al., 2016) }\end{array}$ \\
\hline $\begin{array}{l}\text { Paulownia fortunei var. } \\
\text { mikado }\end{array}$ & $\mathrm{V}, \mathrm{O}$ e $\mathrm{P}$ & $\begin{array}{c}\text { Resultados variados: Stuepp et } \\
\text { al. (20I5a): melhores resultados } \\
\text { no outono. Stuepp et al. (20I5b): } \\
\text { melhores resultados na primavera e } \\
\text { verão. }\end{array}$ & $\begin{array}{l}\text { (STUEPP et al., 20I5a); } \\
\text { (STUEPP et al., 20I5b) }\end{array}$ \\
\hline Pinus taeda $\mathrm{L}$. & Quatro estações & $\begin{array}{l}\text { O período mais favorável foi o } \\
\text { inverno. }\end{array}$ & (ALCANTARA et al., 2007) \\
\hline Platanus acerifolia Ait. & $\mathrm{V}(0 \mathrm{I}), \mathrm{O}(03), \mathrm{I}(07) \mathrm{P}(09)$ & $\begin{array}{l}\text { Melhores resultados durante o } \\
\text { inverno (07). }\end{array}$ & $\begin{array}{l}\text { (NICOLOSO; LAZZARI; } \\
\text { FORTUNATO, I999) }\end{array}$ \\
\hline $\begin{array}{c}\text { Plinia cauliflora (Mart.) } \\
\text { Kausel. }\end{array}$ & $P(10$ e 12$)$ & $\begin{array}{l}\text { Melhores resultados na primavera } \\
\qquad(10) .\end{array}$ & (SILVA et al., 20I9) \\
\hline $\begin{array}{l}\text { Plinia jaboticaba (Vell.) } \\
\text { Berg. }\end{array}$ & $P(10$ e 12$)$ & $\begin{array}{l}\text { Melhores resultados foi a primavera } \\
\qquad(10)\end{array}$ & (SILVA et al., 20I9) \\
\hline $\begin{array}{l}\text { Pongamia pinnata (L.) } \\
\text { Pierre. }\end{array}$ & Revisão & Melhor enraizamento na primavera. & (MUKTA; SREEVALLI, 20I0) \\
\hline
\end{tabular}


Quadro 4 - Resultados que comparam as épocas do ano para diferentes espécies com base nos artigos da revisão bibliográfica sobre estaquia de espécies arbustivo-arbóreas

\begin{tabular}{|c|c|c|c|}
\hline Espécie & Época do ano & Resultados & Referência \\
\hline $\begin{array}{c}\text { Prunus angustifolia } \\
\text { Marshall }\end{array}$ & $\mathrm{P}(05), \mathrm{V}(08), \mathrm{O}(10) * \mathrm{HN}$ & $\begin{array}{l}\text { Melhor enraizamento na primavera } \\
(05) \text { e verão (08) }\end{array}$ & (MCMAHON et al., 20I5) \\
\hline $\begin{array}{l}\text { Prunus persica (L.) } \\
\text { Batsch. }\end{array}$ & Quatro estações & $\begin{array}{c}\text { Melhores resultados na primavera e } \\
\text { verão. }\end{array}$ & $\begin{array}{l}\text { (DUTRA; KERSTEN; } \\
\text { FACHINELLO, 2002) }\end{array}$ \\
\hline $\begin{array}{l}\text { Psidium cattleianum } \\
\text { Sabine }\end{array}$ & $\mathrm{V}, \mathrm{O}$ e I & $\begin{array}{c}\text { A estação do ano mais eficiente foi } \\
\text { o verão. }\end{array}$ & (BIAZATTI et al., 20I8) \\
\hline $\begin{array}{l}\text { Rhododendron simsii } \\
\text { Planch. }\end{array}$ & Quatro estações & $\begin{array}{l}\text { Melhores resultados no fim da } \\
\text { primavera. A época menos favorável } \\
\text { foi o final de inverno. }\end{array}$ & $\begin{array}{c}\text { (FERNANDES; FERNANDES; } \\
\text { MATTOS, 1977) }\end{array}$ \\
\hline $\begin{array}{c}\text { Ricinodendron heudelotii } \\
\text { Baill. }\end{array}$ & $\begin{array}{c}\mathrm{P}(04,05), \mathrm{V}(08,09) \text { e } \mathrm{O} \\
(1 \mathrm{I}, 12) . \mathrm{HN}\end{array}$ & Melhores resultados na primavera. & $\begin{array}{l}\text { (TCHINDA et al., } \\
2013 \text { ) }\end{array}$ \\
\hline $\begin{array}{l}\text { Rosa multiflora cultivar } \\
\text { 'Brooks } 56 \text { '. }\end{array}$ & $\mathrm{I}(02)$ e O (II). *HN & $\begin{array}{l}\text { Melhores resultados no outono (I5 } \\
\text { do } 1 \mid \text { e } 15 \text { do I2). }\end{array}$ & $\begin{array}{l}\text { (HAMBRICK; DAVIES; } \\
\text { PEMBERTON, I99I) }\end{array}$ \\
\hline Rubus spp. & $\mathrm{P}, \mathrm{V}$ e $\mathrm{O}$ & A melhor época foi durante o verão. & (HUSSAIN et al., 20I7) \\
\hline $\begin{array}{l}\text { Sambucus australis } \\
\text { Cham. \& Schltdl. }\end{array}$ & O-I e I-P & $\begin{array}{l}\text { Melhores resultados de } \\
\text { enraizamento no outono-inverno. }\end{array}$ & $\begin{array}{c}\text { (KETTENHUBER; SOUSA; } \\
\text { SUTILI, 20I9) }\end{array}$ \\
\hline $\begin{array}{l}\text { Schefflera arboricola } \\
\text { (Hayata) Merr. }\end{array}$ & $\begin{array}{l}\mathrm{P}(15 \text { e } 25 \text { de } 05) \text { e } V(5 \text { e } \\
\quad 15 \text { de } 07) \text {. *HN }\end{array}$ & $\begin{array}{l}\text { Melhores resultados no verão ( } 15 \\
\text { de } 07) .\end{array}$ & (KHAN, 20I6) \\
\hline $\begin{array}{l}\text { Sesbania. virgata (Cav.) } \\
\text { Pers. }\end{array}$ & O-I e I-P & $\begin{array}{l}\text { Enraizamento somente no outono- } \\
\text { inverno. }\end{array}$ & $\begin{array}{c}\text { (KETTENHUBER; SOUSA; } \\
\text { SUTILI, 20I9) }\end{array}$ \\
\hline $\begin{array}{l}\text { Syringa vulgaris cv. } \\
\text { Charles Joly. }\end{array}$ & $\begin{array}{c}P(05 ; 06) \text { e V }(06) \\
\text { *HN }\end{array}$ & $\begin{array}{l}\text { Melhores resultados na primavera } \\
\qquad(05)\end{array}$ & (CAMERON et al., 2003) \\
\hline $\begin{array}{l}\text { Terminalia australis } \\
\text { Cambess }\end{array}$ & O-I e I-P & Melhores resultados na primavera. & $\begin{array}{c}\text { (KETTENHUBER; SOUSA; } \\
\text { SUTILI, 2019) }\end{array}$ \\
\hline $\begin{array}{l}\text { Tibouchina sellowiana } \\
\text { (Cham.) Cogn. }\end{array}$ & Quatro estações & Melhores resultados no verão. & (FRAGOSO et al., 2017) \\
\hline
\end{tabular}

Legenda: meses indicados pelo numero; P: primavera; V: verão; O: outono; I: inverno; *HN representa trabalhos realizados no hemisfério norte. Os resultados sem marcação foram realizados no hemisfério sul.

Das 40 espécies presentes no Quadro 4, nove apresentaram melhores resultados de enraizamento nas estações de outono e inverno, sendo estas Allamanda cathartica, Campomanesia adamantium, llex paraguariensis, Myrtus communis, Pinus taeda, Platanus acerifolia, Rosa multiflora, Sambucus australis e Sesbania virgata (HAMBRICK; DAVIES; PEMBERTON, I99I; NICOLOSO; LAZZARI; FORTUNATO, I999; KLEIN; COHEN; HEBBE, 2000; ALCANTARA et al., 2007; MARTINS et al., 20I5; STUEPP et al., 20I7; KETTENHUBER; SOUSA; SUTILI, 20I9). Haile (20II), trabalhando com Boswellia papyrifera obtiveram melhores resultados no final do inverno e início da primavera, mas, ao mesmo tempo, os piores resultados foram na primavera, indicando a complexidade da influência da época do ano.

D. brasiliensis foi estudada por Zem em dois momentos diferentes (20I5a; 20I5b). No primeiro trabalho, comparando verão e inverno, obtiveram um maior número de raízes no verão (ZEM et al., 20I5a). Já, em um segundo trabalho, comparando as quatro estações do ano, os resultados de enraizamento foram melhores durante o inverno (ZEM et al., 20I5b). Stuepp et al. (20I5a; 20I5b) obtiveram resultados diversos trabalhando com Paulownia. fortunei. Em um estudo o melhor enraizamento foi durante o outono (STUEPP et al., 20I5a), mas em outro trabalho os resultados de enraizamento foram melhores durante a primavera e verão (STUEPP et al., 20I5b). Nota-se assim, a complexidade da estação do ano para o enraizamento.

Utilizando Ficus carica, tanto Souza et al. (2019) quanto Nava et al. (2014) obtiveram melhores resultados no inverno. Vale destacar que Nava et al. (20l4) compararam dois meses de inverno e, mesmo assim, obtiveram diferenças entre os dois momentos de instalação. Paula et al. (2009) também realizaram estudos com Ficus carica mas, à diferença dos trabalhos anteriores, os melhores resultados foram no final de outono, início da primavera e no verão. Olea europea também foi estudada por diversos autores, apresentando resultados di- 
versos nos diferentes estudos. Penso (2016) obteve melhores resultados no outono e final do inverno início da primavera. Oliveira et al. (2009) também obtiveram melhores resultados no outono. Já Porfírio et al. (20l6), ao realizarem uma revisão sobre a espécie, não observaram resultados consistentes, mas concluíram que os melhores resultados tendem a ser durante o verão, o que difere dos dois estudos anteriormente mencionados. É importante destacar que as estações possuem características muito distintas em diferentes regiões, o que pode levar a resultados diversos.

A época do ano está relacionada com diversos fatores fisiológicos que alteram as características endógenas da planta matriz como, por exemplo, os níveis de hormônios e as reservas energéticas (ZUFFELLATO-RIBAS; RODRIGUES, 200I; HARTMANN et al., 20I8). A propagação vegetativa ocorre de maneira diversa nas diferentes estações. Além disso, tem-se o impacto da floração e produção de frutos, que alteram a quantidade de hormônios e a distribuição dos recursos na planta, podendo alterar o enraizamento. Durante a primavera e verão, a síntese de auxina é maior por ser um período de grande crescimento vegetativo (TAIZ; ZEIGER, 2010). Na primavera e no verão, as condições fisiológicas endógenas, como concentrações de auxinas, carboidratos e cofatores do enraizamento, favorecem a indução radicial.

Conforme apresentado no presente estudo, nota-se a importância da avaliação do efeito destes fatores (presença ou ausência de folhas, tipo de estaca e estação do ano) na indução radicial de cada espécie, podendo atuar de forma diferente no enraizamento de uma espécie para outra. Além disso, foi possível constatar que os fatores podem atuar no enraizamento adventício tanto isoladamente como em conjunto com outros fatores no estímulo à emissão de raízes adventícias.

\section{Considerações Finais}

É possível concluir que, pelo conjunto de espécies abordadas, a partir da revisão bibliográfica do estado da arte da propagação vegetativa por estaquia de espécies arbustivo-arbóreas, a maioria das espécies na literatura apresentaram melhores resultados ao utilizarem estacas herbáceas com folhas durante épocas com temperaturas mais elevadas, como primavera e verão. Assim, recomenda-se, de modo geral, a utilização de estacas herbáceas com folhas, realizadas durante os meses de primavera e verão para a propagação por estaquia de espécies arbustivas-arbóreas a fim de obter melhores resultados de enraizamento.

\section{Referências}

ALCANTARA, G. B. de et al. Efeito da idade da muda e da estação do ano no enraizamento de miniestacas de Pinus taeda L. Revista Árvore, v. 31, n. 3, p. 399-404, 2007.

ALVES, E. C. et al. Number of leaflets on rooting of lychee herbaceous cuttings. Ciência Rural, v. 46, n. 6, p. $1003-$ 1006, jun. 2016.

ANDRADE, R. A. D. et al. Advances in the propagation of Rambutan tree. Revista Brasileira de Fruticultura, v. 39, n. 5,18 dez. 2017.

ARAÚJO, E. F. et al. Mini-cutting techique for vegetative propagation of Paratecoma peroba. CERNE, v. 25, n. 3, p. 314-325, jul. 2019.

BABAIE, $\mathrm{H}$. et al. Effect of different concentrations of IBA and time of taking cutting on rooting, growth and survival of Ficus binnendijkii 'Amstel Queen' cuttings. Notulae Scientia Biologicae, v. 6, n. 2, p. 163-166, 10 jun. 2014.

BEYL, C. A.; TRIGIANO, R. N. (ed.). Plant propagation concepts and laboratory exercises. Second edition ed. Boca Raton, FL: CRC Press, Taylor \& Francis Group, 2015.

BIASI, L. A.; COSTA, G. Propagação vegetativa de Lippia alba. Ciência Rural, v. 33, n. 3, p. 455-459, jun. 2003.

BIAZATTI, M. A. et al. Multiplication of Cattley guava by different techniques and variability among genotypes in vigor and rooting. CERNE, v. 24, n. 4, p. 379-386, dez. 2018. 
CAMERON, R. et al. Rooting cuttings of Syringa vulgaris cv. Charles Joly and Corylus avellana cv. Aurea: the influence of stock plant pruning and shoot growth. Trees - Structure and Function, v. I7, n. 5, p. 45 I-462, I set. 2003.

CARPANEZZI, A. A.; CARPANEZZI, O. T. B. Espécies nativas recomendadas para recuperação ambiental no Estado do Paraná, em solos não degradados. Colombo: Embrapa Florestas, 2006.

CIRIELLO, E.; MORI, E. S. Rooting of Guanandi (Calophyllum brasiliense Cambess) cuttings using indole-butyric acid. CERNE, v. 21 , n. 4, p. 64I-648, dez. 2015.

DIAS, P. C. et al. Estaquia e miniestaquia de espécies florestais lenhosas do Brasil. Pesquisa Florestal Brasileira, v. 32 , n. 72, p. 453-462, $28 \mathrm{dez} .2012$.

DIAS, P. C. et al. Resgate vegetativo de árvores de Anadenanthera macrocarpa. CERNE, v. 2I, n. I, p. 83-89, mar. 20 I5.

DUTRA, L. F.; KERSTEN, E.; FACHINELLO, J. C. Época de coleta, ácido indolbutírico e triptofano no enraizamento de estacas de pessegueiro. Scientia Agricola, v. 59, n. 2, p. 327-333, jun. 2002.

FERNANDES, F. M.; FERNANDES, P. D.; MATTOS, J. R. Multiplicação vegetativa de azálea (Rhododendron simsii, Planch). I. Efeito de fito-hormônio e épocas de estaqueamento. Anais da escola superior de agricultura Luiz de Queiroz, v. 34, n. 0, p. III-120, 1977.

FRAGOSO, R. de O. et al. Vegetative rescue and ex vitro system production of Tibouchina sellowiana clonal plants by cutting and mini-cutting. Ciência Rural, v. 47, n. II, 27 nov. 2017.

FRAGOSO, R. O. et al. Maintenance of leaves and indolebutyric acid in rooting of juvenile Japanese Flowering Cherry cuttings. Revista Brasileira de Ciências Agrárias - Brazilian Journal of Agricultural Sciences, v. I0, n. I, p. 97-I0I, 27 mar. 2015.

GONTIJO, T. C. A. et al. Enraizamento de diferentes tipos de estacas de aceroleira utilizando ácido indolbutírico. Revista Brasileira de Fruticultura, v. 25, n. 2, p. 290-292, ago. 2003.

GUIMARÃES, R. N. et al. Vegetative propagation of Pequi (Souari nut) by cutting. Ciência Rural, v. 49, n. $2,2019$.

HAILE, G. et al. Time of Collection and Cutting Sizes Affect Vegetative Propagation of Boswellia Papyrifera (Del.) Hochst through Leafless Branch Cuttings. Journal of Arid Environments, v. 75, n. 9, p. 873-877, set. 201 I.

HAMBRICK, C. E.; DAVIES, F. T.; PEMBERTON, H. B. Seasonal Changes in Carbohydrate/Nitrogen Levels during Field Rooting of Rosa Multiflora 'Brooks 56' Hardwood Cuttings. Scientia Horticulturae, v. 46, n. I-2, p. I37-I46, fev. I991.

HARTMANN, H. T. et al. Plant propagation: principles and practices. Ninth edition ed. NY, NY: Pearson, 20 I8.

HUSSAIN, I. Effect of different environmental condition on different types of Sweet lime cuttings. Pure and Applied Biology, v. 5, n. 2, p. 298-302, 10 jun. 2016.

HUSSAIN, I. et al. Cutting types collected at different seasons on Blackberry multiplication. Revista Brasileira de Fruticultura, v. 39, n. 3, 17 ago. 2017.

KAMALUDDIN, M.; ALI, M. Effects of Leaf Area and Auxin on Rooting and Growth of Rooted Stem Cuttings of Neem. New Forests, v. 12, n. I, p. II-18, jul. 1996.

KETTENHUBER, P. W.; SOUSA, R.; SUTILI, F. Vegetative propagation of Brazilian native species for restoration of degraded areas. Floresta e Ambiente, v. 26, n. 2, 2019.

KHAN, J. Influence of planting time and bud numbers on growth of Schefflera arboricola propagated through cutting. Pure and Applied Biology, v. 5, n. 3, 10 set. 2016. 
KLEIN, J. D.; COHEN, S.; HEBBE, Y. Seasonal Variation in Rooting Ability of Myrtle (Myrtus Communis L.) Cuttings. Scientia Horticulturae, v. 83, n. I, p. 7I-76, jan. 2000.

KY-DEMBELE, C. et al. Clonal Propagation of Pterocarpus Santalinoides L'Hér. Ex DC.: The Effect of Substrate, Cutting Type, Genotype and Auxin. Southern Forests: a Journal of Forest Science, v. 78, n. 3, p. 193-199, 2 jul. 2016.

LEAKEY, R. R. Physiology of vegetative reproduction. In: Encyclopedia of Forest Sciences. 2004.

LEANDRO, R. C.; YUYAMA, K. Enraizamento de estacas de castanha-de-cutia com uso de ácido indolbutírico. Acta Amazonica, v. 38, n. 4, p. 597-60I, dez. 2008.

LIMA, D. M. et al. Capacidade de enraizamento de estacas de Maytenus muelleri Schwacke com a aplicação de ácido indol butírico relacionada aos aspectos anatômicos. Revista Brasileira de Plantas Medicinais, v. I3, n. 4, p. 422-438, 201 I.

LOSS, A. et al. Induction of rooting in cuttings of Malvaviscus arboreus Cav. with different concentrations of indolbutiric acid (IBA). Acta Scientiarum Agronomy, v. 3I, n. 2, p. 269-273, 2009.

MAGNITSKIY, S.; LIGARRETO, G.; LANCHEROS, H. O. Rooting of two types of cuttings of fruit crops Vaccinium floribundum Kunth and Disterigma alaternoides (Kunth) Niedenzu (Ericaceae). Agronomia Colombiana, v. 29, n. 2, 201 I.

MARTINS, W. A. et al. Estaquia e concentração de reguladores vegetais no enraizamento de Campomanesia adamantium. Ciências Agrárias, v. 38, p. 58-64, 2015.

MBIBONG, D. A.; KANMEGNE, G.; FOTSO. Exogenous Auxins and Leaf Area Affect the Rooting of Xylopia Aethiopica (Dunal A. Rich.) Stem Cuttings. Forests, Trees and Livelihoods, v. 28, n. 4, p. 28I-290, 2 out. 2019.

MCMAHON, E. A. et al. Cutting and Seed Propagation of Chickasaw Plum (Prunus Angustifolia ). International Journal of Fruit Science, v. I5, n. 3, p. 3I3-323, 3 jul. 2015.

MOURÃO FILHO, F. DE A. A.; GIRARDI, E. A.; THADEU ZARATE DO COUTO, H. 'Swingle' citrumelo propagation by cuttings for citrus nursery tree production or inarching. Scientia Horticulturae, v. I20, n. 2, p. 207-2। 2, abr. 2009.

MUKTA, N.; SREEVALLI, Y. Propagation Techniques, Evaluation and Improvement of the Biodiesel Plant, Pongamia Pinnata (L.) Pierre-A Review. Industrial Crops and Products, v. 3I, n. I, p. I-I2, jan. 20 I0.

NAVA, G. A. et al. Rooting of hardwood cuttings of Roxo de Valinhos fig (Ficus carica L.) with different propagation strategies. Revista Ceres, v. 6I, n. 6, p. 989-996, dez. 2014.

NEVES, T. dos S. et al. Enraizamento de corticeira-da-serra em função do tipo de estaca e variações sazonais. Pesquisa Agropecuária Brasileira, v. 4I, n. 12, p. 1699-1705, dez. 2006.

NICOLOSO, F. T.; LAZZARI, M.; FORTUNATO, R. P. Propagação vegetativa de Platanus acerifolia Ait.: (I) efeito de tipos fisiológicos das estacas e épocas de coleta no enraizamento de estacas. Ciência Rural, v. 29, n. 3, p. 479-485, 1999.

OLIVEIRA, A. F. de et al. Estaquia de oliveira em diferentes épocas, substratos e doses de AIB diluído em $\mathrm{NaOH}$ e álcool. Ciência e Agrotecnologia, v. 33, n. I, p. 79-85, fev. 2009.

OLIVEIRA, M. C. de; RIBEIRO, J. F. Enraizamento de estacas de Euplassa inaequalis (Pohl) Engl. de mata de galeria em diferentes estações do ano. Bioscience Journal, v. 29, n. 4, p. 99I-999, 2013.

PAIVA, E. P. de et al. Growth and quality of Mollar pomegranate tree seedlings propagated by cuttings. Semina: Ciências Agrárias, v. 36, n. 6, p. 3629, 9 dez. 2015.

PAULA, L. A. de et al. Efeito do ácido indolbutírico e épocas de estaqueamento sobre o enraizamento de estacas herbáceas de figueira (Ficus carica L.). Acta Scientiarum. Agronomy, v. 31, n. I, p. 87-92, 19 mar. 2009.

PENSO, G. A. et al. Propagação de oliveira “Koroneiki” pelo método de estaquia em diferentes épocas, concentrações de AIB e presença de folhas. Revista Ceres, v. 63, n. 3, p. 355-360, jun. 2016. 
PIO, R. et al. Enraizamento de diferentes tipos de estacas de oliveira (Olea europaea L.) utilizando ácido indolbutírico. Ciência e Agrotecnologia, v. 29, n. 3, p. 562-567, jun. 2005.

PORFÍRIO, S. et al. Reviewing Current Knowledge on Olive (Olea Europaea L.) Adventitious Root Formation. Scientia Horticulturae, v. 198, p. 207-226, jan. 2016.

PURI, S.; VERMA, R. C. Vegetative Propagation of Dalbergia Sissoo Roxb. Using Softwood and Hardwood Stem Cuttings. Journal of Arid Environments, v. 34, n. 2, p. 235-245, out. 1996.

RAMOS-PALACIOS, R. et al. Vegetative propagation of native species potentially useful in the restoration of Mexico City's vegetation. Revista Mexicana de Biodiversidad, v. 83, n. 3, 4 out. 2012.

RINALDI, A. R. et al. Stem cuttings and substrates in Dovyalis asexual propagation. Comunicata Scientiae, v. 8, n. 4, p. 587-595, 12 abr. 2018.

SANTOS, F. M. et al. Produção de mudas de Aloysia gratissima (Gillies \& Hook.) Tronc. por meio da propagação sexuada e assexuada. Revista Brasileira de Plantas Medicinais, v. I I, n. 2, p. I 30-I36, 2009.

SANTOS, M. R. A. D. et al. Estudos sobre superação de dormência em sementes de Smilax japecanga Grisebach. Ciência Agrotecnologia, v. 27, n. 2, p. 319-324, 2003.

SARMIENTO, A. I. P.; SOUZA, P. V. D. de; SCHWARZ, S. F. Collection season and auxin treatment in the propagation by cuttings of mandarin hybrids. Pesquisa Agropecuária Tropical, v. 46, n. 2, p. 2I5-22I, jun. 2016.

SILVA, J. A. A. da et al. Advances in the propagation of Jabuticaba tree. Revista Brasileira de Fruticultura, v. 4I, n. 3, p. e-024, 2019.

SINGH, B.; RAWAT, J. M. S. Effects of Cutting Types and Hormonal Concentration on Vegetative Propagation of Zanthoxylum Armatum in Garhwal Himalaya, India. Journal of Forestry Research, v. 28, n. 2, p. 4I9-423, mar. 2017.

SOLIS, R. et al. Vegetative propagation of Plukenetia polyadenia by cuttings: effects of leaf area and indole-3-butyric acid concentration. Brazilian Journal of Biology, v. 77, n. 3, p. 580-584, 3 out. 2016.

SOUSA, C. M. et al. Effects of Auxin and Misting on the Rooting of Herbaceous and Hardwood Cuttings from the Fig Tree. Revista Ciência Agronômica, v. 44, n. 2, p. 334-338, jun. 2013.

SOUZA, J. M. A. et al. Carbohydrate content and season collection of cuttings from 'Roxo de Valinhos' fig tree. Comunicata Scientiae, v. I0, n. I, p. I25-131, I7 abr. 2019.

SPANDRE, P. et al. Estaquia caulinar de guaçatonga (Casearia sylvestris Swartz) nas quatro estações do ano, com aplicação de diferentes concentrações de AIB. Revista Brasileira de Plantas Medicinais, v. I4, n. 3, p. 529-536, 20I 2.

STUEPP, C. A. et al. Leaf presence and indolebutyric acid on cuttings rooting of dragon tree. Comunicata Scientiae Horticultural Journal, v. 6, n. 2, p. I8I-193, $2015 a$.

STUEPP, C. A. et al. Estaquia de árvores adultas de Paulownia fortunei var. mikado a partir de brotações epicórmicas de decepa. Ciência Florestal, v. 25, n. 3, p. 667-677, 30 set. 20I5b.

STUEPP, C. A. et al. Age of stock plants, seasons and IBA effect on vegetative propagation of Ilex paraguariensis. Revista Árvore, v. 4I, n. 2, 2017.

STUEPP, C. A. et al. Vegetative propagation and application of clonal forestry in Brazilian native tree species. Pesquisa Agropecuária Brasileira, v. 53, n. 9, p. 985-1002, set. 2018.

TAIZ, L.; ZEIGER, E. Fisiologia vegetal. Porto Alegre: Artmed, 2010.

TCHINDA, N. D. et al. Improving Propagation Methods of Ricinodendron heudelotti Baill. from Cuttings. South African Journal of Botany, v. 88, p. 3-9, set. 2013. 
THOMPSON, W. K. Effects of Origin, Time of Collection, Auxins and Planting Media on Rooting of Cuttings of Epacris Impressa Labill. Scientia Horticulturae, v. 30, n. I-2, p. 127-134, nov. 1986.

VELOZA, C. et al. Rooting Ability of Stem Cuttings of Macleania Rupestris Kunth A.C. Sm., a South American Fruit Species. International Journal of Fruit Science, v. I4, n. 4, p. 343-36I, 2 out. 2014.

VIGNOLO, G. K. et al. Presença de folhas no enraizamento de estacas de amoreira-preta. Ciência Rural, v. 44, n. 3, p. 467-472, mar. 2014.

WENDLING, I.; STUEPP, C. A.; ZUFFELLATO-RIBAS, K. C. Araucaria clonal forestry: types of cuttings and mother tree sex in field survival and growth. Cerne, v. 22, n. I, p. 19-26, mar. 2016.

XAVIER, A. Silvicultura clonal princípios e técnicas. Viçosa, MG: Ed. UFV, 2009.

ZEM, L. M. et al. Estaquia Caulinar Herbácea e Semilenhosa de Drimys Brasiliensis. Revista Ciência Agronômica, v. 46, n. 2, 20I5a.

ZEM, L. M. et al. Rooting of semihardwood cuttings of Cataia collected in four seasons. Ciência Rural, v. 45, n. 10, p. $1815-1818,10$ jul. 2015b.

ZEM, L. M. et al. Rooting of semi-hardwood stem cuttings from current year shoots of Drymis brasiliensis. Ciência Rural, v. 46, n. 12, p. 2129-2134, dez. 2016.

ZHANG, W. et al. Mechanisms Underlying the Regulation of Root Formation in Malus Hupehensis Stem Cuttings by Using Exogenous Hormones. Journal of Plant Growth Regulation, v. 36, n. I, p. I74-185, 2016.

ZUFFELLATO-RIBAS, K. C.; RODRIGUES, J. D. Estaquia: uma abordagem dos principais aspectos fisiológicos. Curitiba: [K. C. Zuffellato-Ribas], 200I, 39p. 